\title{
SCIENTIFIC REPORTS

\section{OPEN Assessment of the VDW interaction converting DMAPS from the thermal-motion form to the hydrogen-bonded form}

Received: 26 February 2019

Accepted: 23 August 2019

Published online: 11 September 2019

\author{
Masae Takahashi ${ }^{1}$, Hiroshi Matsui $\mathbb{D}^{2}$, Yuka lkemoto ${ }^{3}$, Makoto Suzuki ${ }^{4}$ \& Nobuyuki Morimoto ${ }^{4}$
}

Assessment of van der Waals (VDW) interactions is fundamental to all of the central quest of structure that regulates the biological function. VDW interactions contributing to intramolecular weak hydrogen bonding are regarded as an important force to regulate the thermal stimuli-sensitive function of sulfobetaine methacrylate, DMAPS. We present here the conversion from the thermal-motion form at room temperature to the weak-hydrogen-bonded form against thermal motion as a terahertz spectral change with a definite isosbestic point from an absorption peak of one form to the other. Vibrational absorptions are used as a probe for assessing VDW interactions in conjunction with highly reliable and well-established density functional theory (DFT) calculations for analysis. Complicated spectral features and uncertain conformations of DMAPS in the amorphous state are clearly resolved under the polarizable continuum model and the dispersion correction for the pure DFT calculations.

The van der Waals (VDW) interaction has become a hot topic in recent years as a constructing force of promising materials such as the VDW heterostructure ${ }^{1-4}$, quantum liquid ${ }^{5,6}$, the VDW bonded magnet ${ }^{7}$, molecular diode ${ }^{8}$, and Rydberg gas 9 . The VDW interaction named in honour of van der Waals, who first introduced attractive interactions between neutral molecules in his equation of state, is a dispersion interaction of pure quantum physical origin $^{10-14}$. The VDW force is well known as attractive at smaller molecular separations, while the sign of the VDW force can be changed from attractive to repulsive by suitable choice of interacting materials immersed in a fluid at larger distances ${ }^{15}$. The VDW forces are very weak and easily disturbed by the thermal energy around room temperature. In biological systems that function at room temperature, VDW interactions provide fantastic characteristics such as fuzzy control in the function of biomolecules. It is significant to directly assess the VDW interactions. How do we determine the efficiency of a VDW interaction? The influence of curvature strain and VDW force on the interlayer vibrational mode of $\mathrm{WS}_{2}$ nanotubes was reported as a redshift by $2.5 \mathrm{~cm}^{-1}$ with confocal micro-Raman spectroscopy ${ }^{16}$. The vibration mode is a promising probe for assessing VDW interactions and can be used in various materials, including biological systems. In particular, terahertz (THz) vibration is powerful for detecting weak interactions ${ }^{17-19}$. Combined with highly reliable and well-established density functional theory (DFT) calculations for the analysis of vibrational absorption spectra, the effect of dispersive interactions can be directly revealed by the observation of vibrational spectral change at low temperature where thermal disturbance is suppressed and the VDW interaction is effective against thermal motion.

The polymerized sulfobetaine methacrylate exhibits a thermal stimuli-responsive function where intramolecular VDW interactions have a decisive role. A sulfobetaine methacrylate, DMAPS (3-[dimethyl-(2-methacryloyloxyethyl) ammonium] propane sulfonate) studied here, is an electrically neutral zwitterionic betaine monomer used in the synthesis of biocompatible polysulfobetaines. The development of biocompatible nanoparticles using zwitterionic polymers is increasingly demanded ${ }^{20-24}$, and we have investigated several biocompatible nanoparticulate drug delivery systems ${ }^{25,26}$. The biocompatibility and the thermal stimuli-responsive function of polysulfobetaines is stemmed from the zwitterionic sulfobetaine monomer. Betaine is a specific type of electrically neutral zwitterion that contains a pair of cationic functional group bearing no hydrogen and anionic functional group at non-adjacent position in the

${ }^{1}$ Graduate School of Agricultural Science, Tohoku University, Sendai, 980-8572, Japan. ${ }^{2}$ Graduate School of Science, Tohoku University, Sendai, 980-8578, Japan. ${ }^{3}$ Japan Synchrotron Radiat. Res. Inst. JASRI SPring-8, Sayo, Hyogo, 679-5198, Japan. ${ }^{4}$ Graduate School of Engineering, Tohoku University, Sendai, 980-8579, Japan. Correspondence and requests for materials should be addressed to M.T. (email: masae.takahashi.d1@tohoku.ac.jp) 


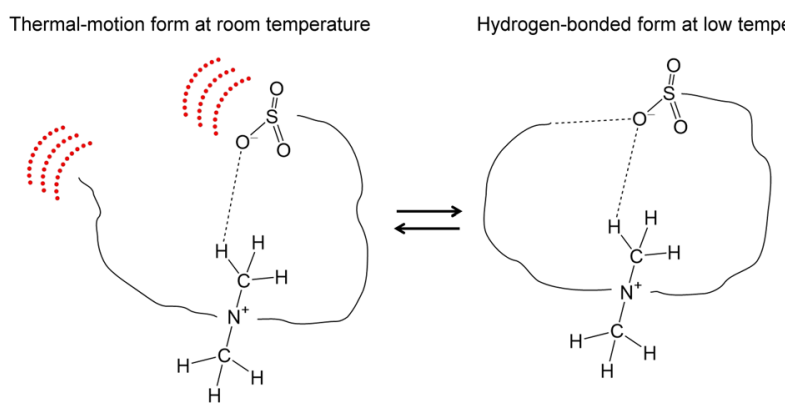

Figure 1. Thermal-motion form of DMAPS at room temperature and hydrogen-bonded form at low temperature.

molecule. It is noteworthy that betaine is in an important position to explore the correlation between the structure and function in biological systems, because the special zwitterionic structure of betaine is biomimic of fundamental biomolecules such as amino acids, proteins, and phospholipids.

$\mathrm{THz}$ peaks from the methacryloyl moiety of DMAPS are predicted to be observed even in the amorphous state $^{27}$. The molecular size of DMAPS is small enough to calculate with reasonable accuracy and sufficient level. DMAPS contains intramolecular weak hydrogen bonds predominantly governed by dispersion interactions such as the VDW force, and the formation of the intramolecular weak hydrogen bonds at low temperature is expected to affect the $\mathrm{THz}$ vibrations. Amorphous samples are more informative from the biological point of view and the $\mathrm{THz}$ spectroscopic study of amorphous samples is desired. However, the study of amorphous samples is lagging behind. A small molecule in an amorphous state gives no spectral feature in the $\mathrm{THz}$ spectral region, although a molecule in a crystalline state gives $\mathrm{THz}$ peaks ${ }^{28}$. Early $\mathrm{THz}$ spectroscopic studies of proteins provided THz peaks even in noncrystalline states ${ }^{29}$, but the peak assignment of proteins is difficult due to the large molecular size. Another issue in the $\mathrm{THz}$ spectroscopic study of amorphous system is the treatment of surrounding molecules in the calculation for peak assignments. We have studied intermolecular weak hydrogen bonding of crystalline compounds using THz spectroscopy with DFT calculations ${ }^{30-33}$ and synchrotron FTIR microspectroscopy ${ }^{34}$. Nowadays, $\mathrm{THz}$ spectroscopic studies are well established for crystalline systems including microcrystalline powder samples and $\mathrm{THz}$ peaks due to intermolecular weak hydrogen bonding networks are obtained as coupled with intermolecular translation and rotation modes. THz peaks are very sensitive to the environment of respective molecule, and even the assignment of intramolecular modes in crystalline compounds requires periodic solid-state calculations that include the effects of surrounding molecules in crystals.

To assess the VDW interaction, we study herein the low temperature behaviour of the biocompatible DMAPS molecule in the vibration absorption of THz spectroscopy and synchrotron FTIR microspectroscopy. Weak hydrogen bonds found in DMAPS are one of three classes of hydrogen bonds ranging from weak (VDW limit) to strong (covalent-bond limit) depending on strength ${ }^{35}$, and the dispersion forces that contribute mainly to the weak hydrogen bond are one of three interactions (electrostatic, induction, and dispersion) contributing to hydrogen bonding. Dispersive interactions, such as VDW interactions and weak hydrogen bonds, are easily disturbed by thermal energy near room temperature (thermal-motion form in Fig. 1), whereas thermal disturbance is suppressed at low temperature, where weak hydrogen bonds are formed against thermal motion (hydrogen-bonded form in Fig. 1). Here we focus on the very weak hydrogen bond formation (hydrogen-bonded form) and cleavage (thermal-motion form) at the VDW limit, where the thermal-motion forms dominate at room temperature. Some hydrogen bonds remain in both hydrogen-bonded and thermal-motion forms. The spectral change in vibrational absorption is predicted to be a change between the thermal-motion and hydrogen-bonded forms. We perform a gas-phase calculation for peak assignment instead of a solid-state calculation because the sample studied here is fully amorphous, Here, we incorporate the dielectric constant into the gas-phase calculation as the effect of the surrounding molecules with a large dipole moment, and perform the dispersion correction for the pure DFT calculation to describe the weak hydrogen bond.

\section{Results}

Low-temperature behaviour of DMAPS in the far-infrared spectrum. The far-infrared (FIR) spectral profile of DMAPS in the range from 140 to $700 \mathrm{~cm}^{-1}$ was measured by synchrotron FTIR microspectroscopy to investigate the behaviour at low temperature where VDW interactions become effective. The overall spectral profile hardly changes upon cooling from room temperature to $4 \mathrm{~K}$, except for peak sharpening and a slight peak shift to high frequency. The spectra from 140 to $700 \mathrm{~cm}^{-1}$ at $150 \mathrm{~K}$ and $4 \mathrm{~K}$ are shown in Fig. 2. Remarkable difference between the two spectra was observed at approximately $300 \mathrm{~cm}^{-1}$. One peak at $150 \mathrm{~K}$ splits in two at 307 and $314 \mathrm{~cm}^{-1}$ at $4 \mathrm{~K}$ (see the square box in Fig. 2), presumably indicating the structural change between the thermal-motion form and hydrogen-bonded form shown in Fig. 1. The insert of Fig. 2 shows the details of the temperature dependence around $300 \mathrm{~cm}^{-1}$ from room temperature to $4 \mathrm{~K}$. It can be seen from the insert of Fig. 2 that the peak splitting occurs clearly below $100 \mathrm{~K}$. It is found from the temperature dependence of the full width at half maximum (FWHM) that the FWHM suddenly decreases at $100 \mathrm{~K}\left(14.5 \mathrm{~cm}^{-1}\right)$ to $150 \mathrm{~K}$ $\left(12.6 \mathrm{~cm}^{-1}\right)$ (Supplementary Fig. S1). Thus, temperature-dependent spectral changes are judged not as thermal broadening but as peak splitting. The thermal-motion form is dominant at $150 \mathrm{~K}$ or more as characterized by one peak and the hydrogen-bonded form dominant at $100 \mathrm{~K}$ or less as characterized by two peaks. A reversible 


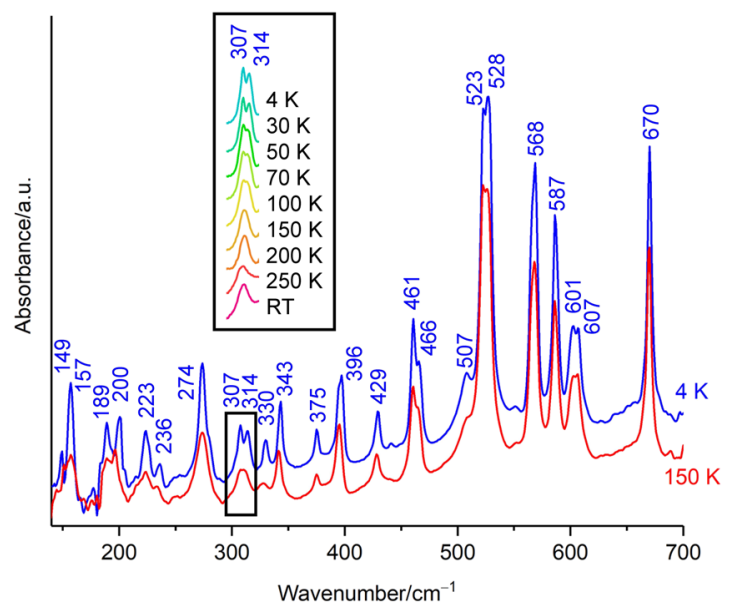

Figure 2. Far-infrared spectrum of DMAPS at $4 \mathrm{~K}$ in blue and $150 \mathrm{~K}$ in red. The temperature dependence at approximately $300 \mathrm{~cm}^{-1}$ from room temperature (RT) to $4 \mathrm{~K}$ indicated by the square box is given in detail in the insert. The frequency of each peak measured at $4 \mathrm{~K}$ is given in $\mathrm{cm}^{-1}$.

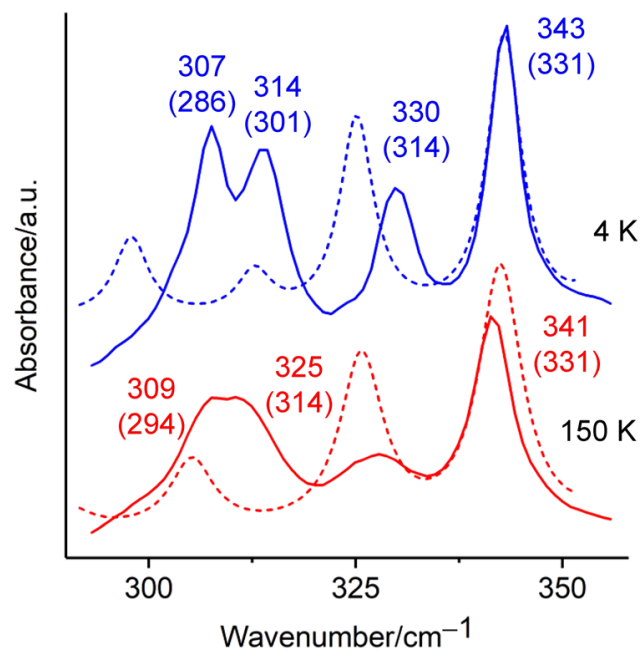

Figure 3. Experimental far-infrared spectra of DMAPS at $150 \mathrm{~K}$ and $4 \mathrm{~K}$ around $300 \mathrm{~cm}^{-1}$ (solid lines) and calculated spectra using dispersion-corrected DFT under the PCM model with dielectric constants $(\varepsilon)$ of 15.20 (red dashed line) and 8.33 (blue dashed line). The FWHM of the calculated spectrum is set to the same value as that of the strongest peak in this frequency region $\left(6.4\right.$ and $5.0 \mathrm{~cm}^{-1}$, respectively). The calculated spectrum is shifted by $12 \mathrm{~cm}^{-1}$ so that the strongest peaks of the blue dashed and solid lines coincide. The experimental and calculated frequencies (in parentheses) of respective peak are given in $\mathrm{cm}^{-1}$.

temperature-dependent spectral change was obtained between the cooling process from room temperature to $4 \mathrm{~K}$ and the heating process from $4 \mathrm{~K}$ to room temperature. It is well known that conformational differences are distinguished from each other in the FIR frequency region. The various conformations possible for DMAPS showed considerably different spectral patterns in our DFT calculations. Therefore, no remarkable spectral change from room temperature to $4 \mathrm{~K}$ suggests no conformational change due to cooling. In addition, the possibility of a conformational mixture or a dimer formation is excluded due to the number and sharpness of the measured peaks, although the conformation in the amorphous powder of DMAPS is unknown.

Figure 3 shows the calculated FIR spectra together with the experimental ones around $300 \mathrm{~cm}^{-1}$ where the temperature-dependent peak splitting was experimentally observed in Fig. 2. The calculated FIR spectra in Fig. 3 are those of the conformer giving the best-fit FIR spectrum to the experimental $4 \mathrm{~K}$ result both in frequency and intensity, among the spectra of stable conformations in Gibbs free energy at $298 \mathrm{~K}$ within the $2 \mathrm{kcal} / \mathrm{mol} \mathrm{accu}-$ racy of highly accurate CBS-Q (see Supplementary Table S1 for thermodynamic data). The second most stable conformer was selected as that giving the best-fit FIR spectrum (the optimized dihedral angles representing the selected conformation are given in Fig. 4). The spectrum calculated under the polarizable continuum (PCM) model ${ }^{36}$ with a dielectric constant of 8.33 excellently reproduces four peaks around $300 \mathrm{~cm}^{-1}$ (see the blue solid and dashed lines in Fig. 3): 307, 314, 330, and $343 \mathrm{~cm}^{-1}$ (observation at $4 \mathrm{~K}$ ); 286, 301, 314, and $331 \mathrm{~cm}^{-1}$ (calculation with a dielectric constant of 8.33). The discrepancy between the calculated frequency and the experimental 


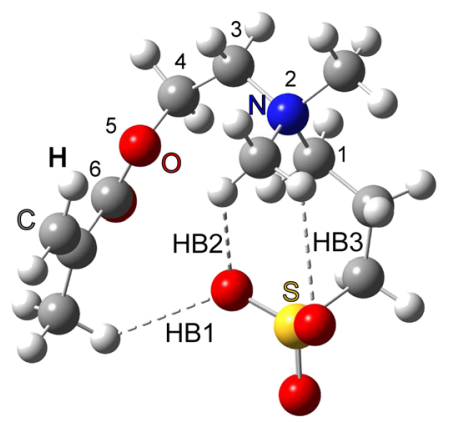

Dihedral angle

$d(1,2,3,4): 46.7^{\circ}(\varepsilon=8.33)$

$46.2^{\circ}(\varepsilon=15.20)$

$d(2,3,4,5): 62.8^{\circ}(\varepsilon=8.33)$

$62.7^{\circ}(\varepsilon=15.20)$

$d(3,4,5,6):-151.3^{\circ}(\varepsilon=8.33)$

$-151.1^{\circ}(\varepsilon=15.20)$

Figure 4. DMAPS conformer giving the best-fit FIR spectrum under the PCM model at the B3LYP/aug-cc$\mathrm{pVTZ}$ level with dispersion correction. The atoms characterising the selected conformation are numbered from 1 to 6 , and their dihedral angles at $\varepsilon=8.33$ and 15.20 are shown. HB1, HB2, and HB3 are the O...H hydrogen bonds showing remarkable differences by changing the dielectric constant.

\begin{tabular}{|c|c|c|c|c|c|c|c|c|}
\hline $\mathbf{N}$ & $\varepsilon$ & Solvent & HB1/Å & HB2/Å & HB3/Å & $\begin{array}{l}\nu_{1} / \\
\mathrm{cm}^{-1}\end{array}$ & $\begin{array}{l}\nu_{2} / \\
\mathrm{cm}^{-1}\end{array}$ & $\begin{array}{l}\nu_{3} / \\
\mathrm{cm}^{-1}\end{array}$ \\
\hline \multirow{3}{*}{3} & 46.83 & Dimethyl sulfoxide & 2.93 & 2.57 & 3.05 & $16(3)$ & $27(8)$ & $36(18)$ \\
\hline & 20.49 & Acetone & 2.83 & 2.53 & 2.93 & $23(5)$ & $26(6)$ & $41(13)$ \\
\hline & 15.20 & 2-Pentanone & 2.79 & 2.51 & 2.89 & $31(8)$ & $35(6)$ & $43(16)$ \\
\hline \multirow{3}{*}{4} & 10.13 & Dichloroethane & 2.77 & 2.48 & 2.82 & $35(11)$ & $43(8)$ & $48(9)$ \\
\hline & 8.93 & Dichloromethane & 2.77 & 2.47 & 2.80 & $34(11)$ & $44(7)$ & $49(8)$ \\
\hline & 8.33 & Ethyl methanoate & 2.77 & 2.46 & 2.79 & $33(11)$ & $43(6)$ & $49(9)$ \\
\hline \multirow{3}{*}{5} & 2.91 & Dipropylamine & 2.56 & 2.45 & 2.42 & $33(7)$ & $55(9)$ & $56(3)$ \\
\hline & 2.23 & Carbon tetrachloride & 2.54 & 2.41 & 2.39 & $18(7)$ & $49(1)$ & $57(10)$ \\
\hline & 1.43 & Argon & 2.49 & 2.38 & 2.27 & $25(5)$ & $47(5)$ & $55(5)$ \\
\hline
\end{tabular}

Table 1. Effects of dielectric constants $(\varepsilon)$ on the number of peaks around $300 \mathrm{~cm}^{-1}(\mathrm{~N})$, bond distances of intramolecular hydrogen bonds (HB1, HB2, and HB3), and vibrational absorption frequencies of the first three vibrations $\left(\nu_{1}, \nu_{2}, \nu_{3}\right)$ calculated at the B3LYP/aug-cc-pVTZ with dispersion correction. For three intramolecular hydrogen bonds, HB1, HB2, and HB3, see Fig. 4. The IR intensities are given in $\mathrm{km} / \mathrm{mol}$ in parentheses. Solvent keywords in SCRF $=$ Solvent option are indicated as solvent.

value of Fig. 2 is small enough (less than $10 \mathrm{~cm}^{-1}$ ) in the range of $140-250 \mathrm{~cm}^{-1}$, while the deviation of the calculated value from experimental result in the range of $250-700 \mathrm{~cm}^{-1}$ is relatively large and always negative (up to $32 \mathrm{~cm}^{-1}$ ) (see Supplementary Table S2). This is probably a hypsochromic shift due to packing and/or repulsive interactions with surrounding molecules. The temperature-dependent spectral change around $300 \mathrm{~cm}^{-1}$ from four peaks at $4 \mathrm{~K}$ to three peaks at $150 \mathrm{~K}$ is reproduced by changing the dielectric constant from 8.33 to 15.20 in the PCM model (dashed lines in Fig. 3). The difference of the dielectric constant affects scarcely the spectral profile in the whole range of $140-700 \mathrm{~cm}^{-1}$ except for the two peaks. It is reasonable that the spectrum of higher dielectric constant (15.20) exhibits the higher temperature spectrum, because the increase of temperature raises the dielectric constant due to the higher mobility of molecules caused by the cleavage of hydrogen bond at higher temperatures.

The number of peaks near $300 \mathrm{~cm}^{-1}(\mathrm{~N})$ changes from 5 to 3 as the dielectric constant increases (Table 1). $\mathrm{N}=4$ and 3 correspond to the spectra below $100 \mathrm{~K}$ (low-temperature model) and above $150 \mathrm{~K}$ (room-temperature model), respectively. The spectral features (frequency and intensity) around $300 \mathrm{~cm}^{-1}$ are almost the same when $\mathrm{N}$ is the same. On the other hand, the frequencies of $\nu_{1}, \nu_{2}$, and $\nu_{3}$ in the $\mathrm{THz}$ spectral region hardly change when $\mathrm{N}=4$, but decrease with the increase of the dielectric constant when $\mathrm{N}=3$. Since the frequencies of $\nu_{1}$ are closest to each other and reproduce the stationary features of $\nu_{1}$ in the THz spectrum, ethyl methanoate $(\varepsilon=8.33)$ and 2-pentanone $(\varepsilon=15.20)$ are selected as solvents for the low-temperature model below $100 \mathrm{~K}$ and high-temperature model above $150 \mathrm{~K}$, respectively.

Structural difference with the difference of dielectric constant. Full geometry optimization was performed for the conformer giving the best-fit FIR spectrum to the experimental $4 \mathrm{~K}$ result, using a different dielectric constant, $\varepsilon$, under the PCM model at the B3LYP/aug-cc-pVTZ level with dispersion correction. Among 27 conformations for atoms 1-6 in Fig. 4, we obtained 24 conformers. The optimized geometric parameters hardly change between different dielectric constants except for three hydrogen bond distances, HB1, HB2, and HB3 (see Fig. 4). The conformations of the two optimized structures used as low-temperature model $(\varepsilon=8.33)$ and high-temperature model $(\varepsilon=15.20)$ are the same (see the dihedral angles of two models in Fig. 4). The different bonding nature of $\mathrm{HB} 1$ depending on the $\mathrm{N}$ value indicates that $\mathrm{HB} 1$ plays a decisive role in controlling the number of peaks around $300 \mathrm{~cm}^{-1}$ (Table 1). At the lowest three $\varepsilon^{\prime} s$ where $\mathrm{N}=5$, the distance is the typical $\mathrm{O}$... H distance between the $\mathrm{CH}$ donor and the $>\mathrm{S}=\mathrm{O}$ accepter $(2.49 \sim 2.56 \AA)^{37}$. The distance gradually increases 

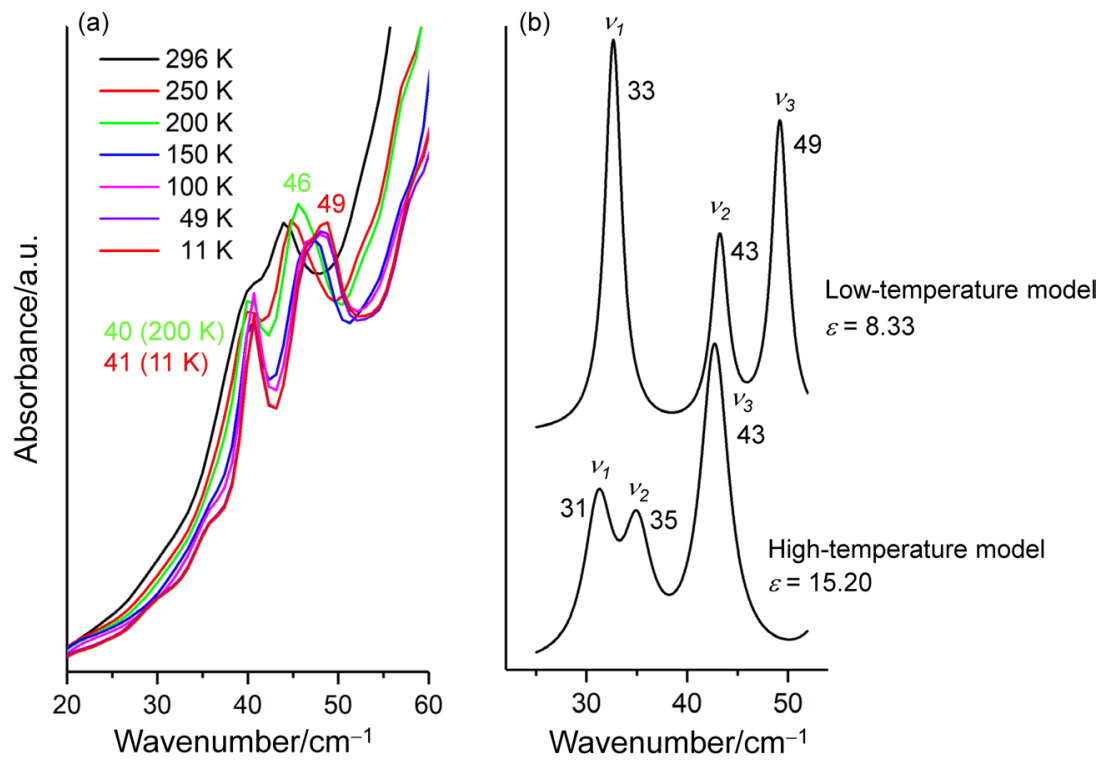

Figure 5. (a) Temperature-dependent THz spectra of DMAPS and (b) the calculated THz spectra of the first three vibrations $\left(\nu_{1}, \nu_{2}, \nu_{3}\right)$ using the different dielectric constants $(\varepsilon)$ under the PCM model at the dispersioncorrected B3LYP/aug-cc-pVTZ level. The FWHM of the calculated spectrum is set to the same value as that of the lowest peak $\left(2.0 \mathrm{~cm}^{-1}\right.$ for $\varepsilon=8.33$ and $3.4 \mathrm{~cm}^{-1}$ for $\varepsilon=15.20$, respectively). The calculated and the observed frequencies are given in $\mathrm{cm}^{-1}$.

and its bond strength weakens as the dielectric constant increases and the $\mathrm{O}$ atom accepter basicity decreases. At the highest three $\varepsilon^{\prime} s$ where $\mathrm{N}=3$, the distance is much more than VDW contact $(>2.79 \AA)^{38}$ and no hydrogen bonding exists. At the middle three $\varepsilon^{\prime} s$ where $\mathrm{N}=4, \mathrm{HB} 1$ gives a constant value $(2.77 \AA)$ suggesting VDW contacts probably due to quantum fluctuations. In that sense, HB1 at the middle three $\varepsilon^{\prime} s$ is a weak hydrogen bond at the VDW limit. For all dielectric constants, HB2 gives a typical O...H distance between the $\mathrm{CH}$ donor and the $>\mathrm{S}=\mathrm{O}$ accepter $(2.38 \sim 2.57 \AA)^{37}$, and the permittivity dependence is the same as HB1 at the lowest three $\varepsilon^{\prime}$ s. Even at the middle three $\varepsilon^{\prime} s$ where $\mathrm{N}=4$, the HB3 distance is greater than $2.79 \AA$ and no hydrogen bonds already exist in this dielectric constant range.

THz spectra of DMAPS. The vibrational absorption spectrum in the THz region $\left(20-60 \mathrm{~cm}^{-1}\right)$ shows a clearer temperature-dependent spectral change (Fig. 5a), presumably indicating the structural change between the thermal-motion form and hydrogen-bonded form shown in Fig. 1. A reversible temperature-dependent spectral change was obtained between the cooling process from room temperature to $11 \mathrm{~K}$ and the heating process from $11 \mathrm{~K}$ to room temperature. At room temperature $(296 \mathrm{~K})$, a broad peak with a shoulder was observed (black line in Fig. 5a). By cooling to $200 \mathrm{~K}$, the two peaks sharpen $\left(40 \mathrm{~cm}^{-1}\right.$ and $\left.46 \mathrm{~cm}^{-1}\right)$, and the stronger peak shifts remarkably to higher frequency (green line in Fig. 5a). Peak sharpening and high frequency shifts due to the anharmonicity of the potential energy surface in the cooling process are common in the $\mathrm{THz}$ spectral region $27,33,39-41$. The peak position at $40 \mathrm{~cm}^{-1}$ changes little by further cooling, while the peak at $46 \mathrm{~cm}^{-1}$ moves to $49 \mathrm{~cm}^{-1}$ with an isosbestic point in-between. The intensity of the peak at $49 \mathrm{~cm}^{-1}$ decreases compared to that of the peak at $46 \mathrm{~cm}^{-1}$, leaving a shoulder remaining on the low frequency side of the peak at $49 \mathrm{~cm}^{-1}$.

The spectral change is interpreted as the difference of dielectric constant under the PCM model (Fig. 5b). The calculation with the dielectric constant of 15.20 (high-temperature model) gives three peaks at 31,35 , and $43 \mathrm{~cm}^{-1}$. The frequency and relative intensity excellently agree with the observed spectra at $200 \mathrm{~K}$. That is, the frequency difference is less than $10 \mathrm{~cm}^{-1}$ and $\nu_{3}$ is the strongest peak. Two peaks $\nu_{1}$ and $\nu_{2}$ may have produced one broad peak centred around $33 \mathrm{~cm}^{-1}$. By lowering the dielectric constant to $8.33, \nu_{2}$ and $\nu_{3}$ shift to higher frequency to 43 and $49 \mathrm{~cm}^{-1}$, and $\nu_{1}$ becomes stronger at $33 \mathrm{~cm}^{-1}$ compared with $\nu_{2}$ and $\nu_{3}$ (upper panel of Fig. $5 \mathrm{~b}$ ). The peak $\nu_{2}$ may be the shoulder that remains at $11 \mathrm{~K}$. The spectral change with decreasing the dielectric constant from 15.20 to 8.33 well corresponds the temperature-dependent spectral change from $200 \mathrm{~K}$ to $11 \mathrm{~K}$. Unlike the THz spectrum of polymers ${ }^{42}$, DMAPS studied here is a monomer molecule, so the possibility of partial crystallization like in an amorphous one-dimensional long polymer chain is excluded. An observed background growing monotonically from 20 to $60 \mathrm{~cm}^{-1}$ is the characteristics of amorphous THz spectra ${ }^{28}$.

Vibrational modes giving temperature dependence. The vibrational modes of DMAPS showing a temperature-dependent spectral changes in the FIR and THz regions are examined. The peaks at 307 and $314 \mathrm{~cm}^{-1}$ observed at $4 \mathrm{~K}$ in the FIR region are assigned to the torsion modes, $\tau_{1}\left(-\mathrm{CH}_{3}\right)$ (disrotation of one methyl group and $\mathrm{N}(\mathrm{MeR} 1 \mathrm{R} 2)$ group) and $\tau_{2}\left(-\mathrm{CH}_{3}\right)$ (corotation of two methyl groups on a nitrogen atom) (Fig. 6). As shown in Table 1, HB2 is a typical O...H bond between methyl hydrogen and $\mathrm{SO}_{3}$ oxygen in both the low-temperature model $(\varepsilon=8.33)$ and high-temperature model $(\varepsilon=15.20)$. The strength of HB2 gradually 


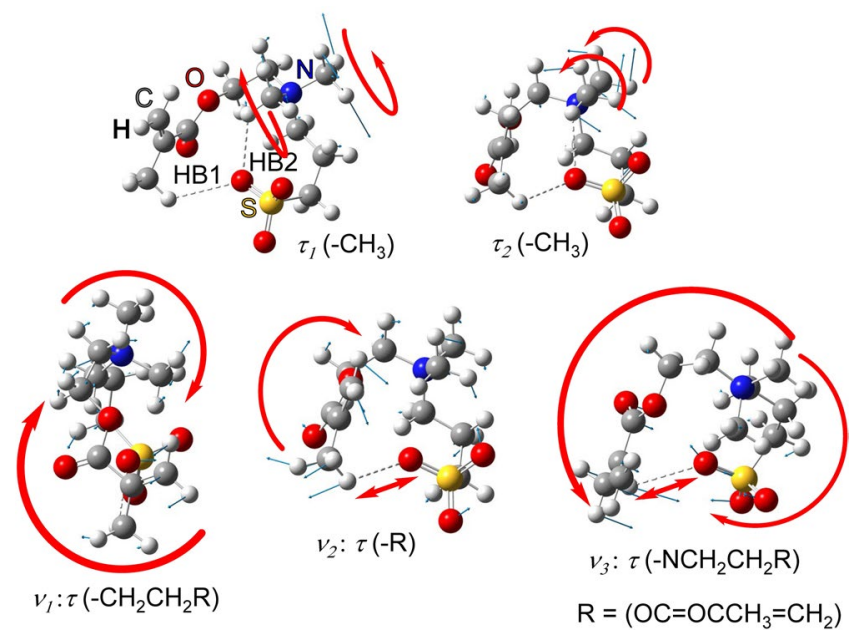

Figure 6. Vibrational mode of two peaks showing a temperature-dependent spectral change in the FIR region of DMAPS $\left(\tau_{1}\left(-\mathrm{CH}_{3}\right)\right.$ and $\left.\tau_{2}\left(-\mathrm{CH}_{3}\right)\right)$ and the lowest three vibrations in $\mathrm{THz}$ region $\left(\nu_{1}, \nu_{2}, \nu_{3}\right)$.

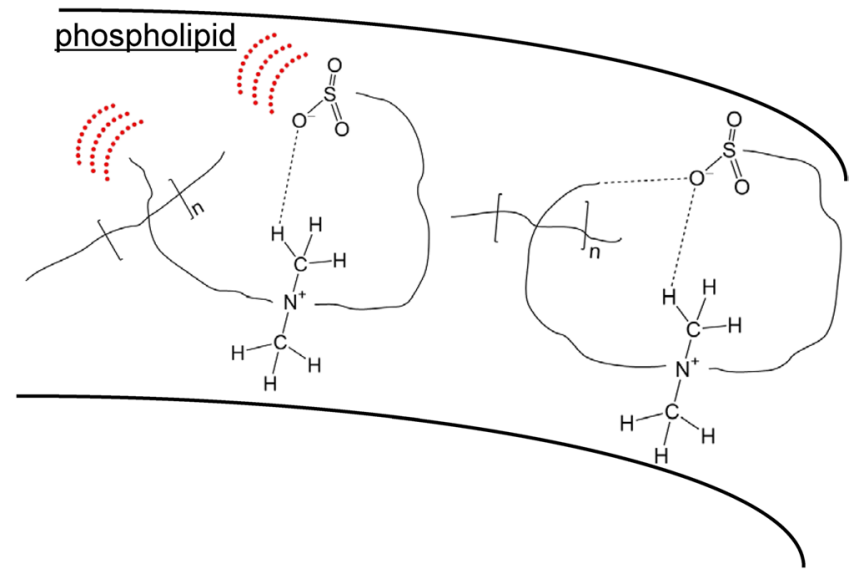

Figure 7. Stimuli-sensitive biocompatible DMAPS polymers that change the form depending on the environment.

decreases as the dielectric constant increases. On the other hand, HB1 makes weak hydrogen bond at the VDW limit in low-temperature model, but not in high-temperature model. In low-temperature mode $(\varepsilon=8.33)$, the frequency and intensity of $\tau_{1}\left(-\mathrm{CH}_{3}\right)$ and $\tau_{2}\left(-\mathrm{CH}_{3}\right)$ increase due to the increase of $\mathrm{HB} 2$ bond strength. Therefore, totally four peaks including $\tau_{1}\left(-\mathrm{CH}_{3}\right)$ and $\tau_{2}\left(-\mathrm{CH}_{3}\right)$ were observed below $100 \mathrm{~K}$. Mode $\tau_{2}\left(-\mathrm{CH}_{3}\right)$ includes movement in the HB1 bonding direction, but not $\tau_{1}\left(-\mathrm{CH}_{3}\right)$. The cleavage of $\mathrm{HB} 1$ at $\varepsilon=15.20$ (high-temperature model) allows the $\mathrm{O}$ atom of the $\mathrm{SO}_{3}$ group to move without constraint from the $\mathrm{HB} 1$ bond, and only the intensity of $\tau_{2}\left(-\mathrm{CH}_{3}\right)$ increases. Thus, totally three peaks were observed above $150 \mathrm{~K}$.

Three peaks observed in the THz spectrum are assigned to three torsion modes $\left(\nu_{1}, \nu_{2}\right.$, and $\nu_{3}$ in Fig. 6). Two of the three modes, $\nu_{2}$ and $\nu_{3}$, contain stretching vibrations of the HB1 hydrogen bond. As shown in Table 1, HB1 forms a weak hydrogen bond at $\varepsilon=8.33$ and not at $\varepsilon=15.20$. Thus, with the formation of HB1 hydrogen bonding at $\varepsilon=8.33$, the frequencies of $\nu_{2}$ and $\nu_{3}$ increase.

\section{Discussion}

Our current work has succeeded in assessing the VDW interaction that converts DMAPS from the thermal-motion form to the hydrogen-bonded form shown in Fig. 1. The conversion between the two forms was obtained clearly as a spectral change in the $\mathrm{THz}$ spectral region with a definite isosbestic point indicating a change from one absorption peak to the other. Temperature-dependent spectral change occurred between 150 and $100 \mathrm{~K}$ which corresponds to nearly the VDW bonding energy $(<240 \mathrm{~K})$. The spectral change by cooling was interpreted as the decrease of dielectric constant due to the reduced mobility at low temperature. Present results suggest that the stimuli-sensitive biocompatible DMAPS polymers change their form depending on the environmental temperature and dielectric constant via the formation of weak hydrogen bond (Fig. 7). The dielectric constants, 8.33. and 15.20 used in the present study are within the range of the dielectric constant of proteins and phospholipids (4 20).

We also succeeded in obtaining distinct THz peaks from completely amorphous DMAPS powder. Most biological materials act in amorphous form, and crystals are rare. Generally, a crystal has a sharp THz peak, and 
the amorphous form has no feature in the THz spectrum. On the other hand, DMAPS studied here gave $\mathrm{THz}$ peaks even though it is not crystalline. It is important to distinguish between the absence of a THz peak and the presence of a very weak and/or broad $\mathrm{THz}$ peak. Our previous $\mathrm{THz}$ spectroscopic study on crystalline sample ${ }^{27}$ has found that the chain torsion mode is present in the THz energy region. Although there is a crystal field effect, it is expected that vibrational absorption by the torsion mode of the methacryloyl moiety exists near this region. The problem is presumed to be a very low intensity of the vibrational absorption. To overcome this problem, we measured the sample without using a filling material such as polyethylene to dilute the sample. Regarding filling materials, there are also noticeable points in the synchrotron FTIR microspectroscopy measurements in our present study. $\mathrm{KBr}$ is normally used as a filling material in FTIR measurements but is not a suitable filling material for DMAPS because $\mathrm{KBr}$ may interact with anion and cation pairs in electrically neutral zwitterionic betaine monomer such as DMAPS. Synchrotron FTIR microspectroscopy requires no filling material for measurements and can avoid this interaction.

The complicated FIR spectrum of the present amorphous system was found to be well reproduced by incorporating the effect of the surrounding molecules as the dielectric constant. Deduced from the dielectric constant of the analogous, we have investigated nine solvents with dielectric constants from 1.43 to 46.83 (Table 1). From the number of peaks around $300 \mathrm{~cm}^{-1}$ and the frequency in the $\mathrm{THz}$ region, we finally selected the value 8.33 for the low-temperature model and 15.20 for the high-temperature model. When incorporating a dielectric constant of 8.33 in the calculation, the calculated spectrum reproduces the experimental spectrum for both frequency and intensity, and the frequency deviation is less than $32 \mathrm{~cm}^{-1}$.

\section{Conclusions}

Based on measurements of the temperature-dependent behavior of amorphous DMAPS in the FIR and THz spectral regions and DFT calculations with dispersion correction, we have assessed the VDW interactions that convert DMAPS from the thermal-motion form to the hydrogen-bonded form. In order to treat VDW interactions very accurately, we used the dispersion correction method that provides the accuracy for weak interactions comparable to $\operatorname{CCSD}(\mathrm{T})$ that can attain a consistently accurate description of VDW interactions. We found a very weak intramolecular hydrogen bond at the VDW limit formed below $100 \mathrm{~K}$, and the contact distance is calculated to be $2.77 \AA$. The formation of very weak hydrogen bonds critically causes a change in the number of peaks around $300 \mathrm{~cm}^{-1}$ in the FIR spectral region and a high frequency shift for certain modes in the THz spectral region. The present finding of very weak intramolecular hydrogen bonds at the VDW limit in a biocompatible compound leads to the elucidation of the role of VDW interactions in the function of thermal stimuli-sensitivity and biocompatibility.

\section{Methods}

Materials. DMAPS (3-[dimethyl (2-methacryloyloxyethyl) ammonium] propane sulfonate) was purchased from Sigma-Aldrich (St. Louis, MO) and measured in both synchrotron FTIR microspectroscopy and terahertz time-domain spectroscopy (THz-TDS) without further purification.

Synchrotron FTIR microspectroscopy. Synchrotron FTIR spectra in the range of $140-700 \mathrm{~cm}^{-1}$ were collected with a resolution of $2 \mathrm{~cm}^{-1}$ at an infrared beamline BL43IR of the SPring- 8 synchrotron radiation (SR) facility (Hyogo, Japan). An FTIR (BRUKER IFS120HR) spectrometer was used with IR-SR as an infrared source. The powder sample dissolved in desalinated water was dropped on a silicon substrate and dried, then inserted into the cryostat and evacuated. The spectrum was the same, even when the powder sample was placed directly on a silicon substrate without dissolving in desalinated water and was inserted into the cryostat and measured. By evacuation it is estimated that the water disappears completely during the measurement. The frequency of each peak in Figs. 2 and 3 was evaluated by fitting the spectrum with a Lorentz function.

THz-TDS. THz spectra from 20 to $60 \mathrm{~cm}^{-1}$ were measured with a Tochigi Nikon RT-20000 THz-TDS with a resolution of $0.8 \mathrm{~cm}^{-1}$ at $11-296 \mathrm{~K}$. Powder samples were placed directly on the sample holder with Kapton tape on both sides. We confirmed that the Kapton tape has no absorption in the $\mathrm{THz}$ spectral region.

DFT calculations. To analyse the vibrational absorption spectrum of a fully amorphous powder sample, DFT calculations of gas-phase molecules surrounded by themselves were performed with the Gaussian 09 and 16 software packages ${ }^{43}$. We utilized a hybrid Becke-type three-parameter exchange functional ${ }^{44}$ paired with the gradient-corrected Lee, Yang, and Parr correlation functional (B3LYP) ${ }^{45,46}$ and the aug-cc-pVTZ basis set ${ }^{47-51}$. The Petersson-Frisch dispersion model from the APDF functional was used for dispersion correction ${ }^{52}$. This model provides comparable weak interaction accuracy to that of $\operatorname{CCSD}(\mathrm{T}) /$ aug-cc-pVTZ, which can attain a consistently accurate description of VDW interactions. The geometric parameters were fully optimized, and the optimized structures were confirmed to have no imaginary frequency. To select stable conformers, the Gibbs free energy at $298 \mathrm{~K}$ was calculated with highly accurate CBS-Q (see Supplementary Table S1 for thermodynamic data). The effect of the surrounding molecules was incorporated as a solvent under the PCM model using the integral equation formalism variant (IEF-PCM) ${ }^{36}$. To investigate the effect of various solvents and their dielectric constants, 9 solvent keywords accepted with SCRF = Solvent option were used. The cavity type used for PCM calculations was a scaled VDW which places a sphere around each solute atom with the universal force field (UFF) radii ${ }^{53}$ scaled by a factor of 1.1 . The scaling factor depends on solvent, solute ${ }^{36}$, and the definition of atomic radii $^{54,55}$. Examining the effects of the scaling factors, we found that the results with factor 1.1 fit well to the FIR spectrum at $4 \mathrm{~K}$ and can account for temperature-dependent spectral changes. To obtain the reliable result for very low frequency as in $\mathrm{THz}$ region, a superfine integration grid was used.

We investigated all possible conformations of the methacryloyl moiety of the DMAPS molecule for the propane sulfonate moiety in the ring form at the B3LYP/cc-pVTZ to elucidate the conformation of DMAPS in the 
amorphous state studied here (see Supplementary Fig. S2). For the propane sulfonate moiety, we confirmed that the ring form connected between positive nitrogen and negative oxygen is much more stable by $18 \mathrm{kcal} / \mathrm{mol}$ than the linear form without $\mathrm{N}^{+}-\mathrm{O}^{-}$connection at the B3LYP/cc-pVTZ + ZPE level with dispersion correction (D3 version of Grimme's empirical dispersion with the original D3 damping function) ${ }^{56}$. We have obtained 24 conformations, starting from 27 conformations of methacryloyl moiety with a transoid $-\mathrm{C}(=\mathrm{O})-\mathrm{C}\left(\mathrm{CH}_{3}=\mathrm{CH}_{2}\right)$ at the terminal.

\section{Data Availability}

The data generated or analysed during this study are available from the corresponding author on reasonable request.

\section{References}

1. Geim, A. K. \& Grigorieva, I. V. Van der Waals heterostructures. Nature 499, 419-425 (2013).

2. Molina-Mendoza, A. J. et al. Franckeite as a naturally occurring van der Waals heterostructute. Nat. Commun. 8, 14409 (2017).

3. Jariwala, D., Marks, T. J. \& Hersam, M. C. Mixed-dimensional van der Waals heterostructures. Nat. Mater. 16, 170-181 (2017).

4. Georgiou, T. et al. Vertical field-effect transistor based on graphene- $\mathrm{WS}_{2}$ heterostructures for flexible and transparent electronics. Nat. Nanotechnol. 8, 100-103 (2013).

5. Petrov, D. S. Liquid beyond the van der Waals paradigm. Nat. Phys. 14, 211-212 (2018).

6. Cabrera, C. R. et al. Quantum liquid droplets in a mixture of Bose-Einstein condensates. Science 359, 301-304 (2018).

7. Liu, B. et al. Critical behavior of the van der Waals bonded high $T_{c}$ ferromagnet $\mathrm{Fe}_{3} \mathrm{GeTe}_{2}$. Sci. Rep. 7, 6184 (2017).

8. Nerngchamnong, N. et al. The role of van der Waals forces in the performance of molecular diodes. Nat. Nanotechnol. 8, 113-118 (2013).

9. Schanß, P. et al. Observation of spatially ordered structures in a two-dimensional Rydberg gas. Nature 491, 87-91 (2012).

10. Kaplan, I. G. Intermolecular interactions: Physical picture, computational methods and model potentials (John Wiley \& Sons, Ltd., 2006).

11. Casimir, H. B. G. \& Polder, D. The influence of retardation on the London-van der Waals forces. Phys. Rev. 73, 360-372 (1948).

12. Buhmann, S. Y. Dispersion Forces I: Macroscopic quantum electrodynamics and ground-state Casimir, Casimir-Polder and van der Waals forces (Springer-Verlag Berlin Heidelberg, 2012).

13. Dalvit, D., Milonni, P., Roberts, D. \& da Rosa, F. Casimir physics (Springer-Verlag Berlin Heidelberg, 2011).

14. Simpson, W. M. R. \& Leonhardt, U. Forces of the quantum vacuum: An introduction to Casimir physics (World Scientific, 2015).

15. Munday, J. N., Capasso, F. \& Parsegian, V. A. Measured long-range repulsive Casimir-Lifshitz forces. Nature 457, 170-173 (2009).

16. Wang, X. H., Zheng, C. C. \& Ning, J. Q. Influence of curvature strain and van der Waals force on the interlayer vibration mode of $\mathrm{WS}_{2}$ nanotubes: A confocal micro-Raman spectroscopic study. Sci. Rep. 6, 33091 (2016).

17. Baxter, J. B. \& Guglietta, G. W. Terahertz spectroscopy. Anal. Chem. 83, 4342-4368 (2011).

18. Ueno, Y. \& Ajito, K. Analytical terahertz spectroscopy. Anal. Sci. 24, 185-192 (2008).

19. Plusquellic, D. F., Siegrist, K., Heilweil, E. J. \& Esenturk, O. Applications of terahertz spectroscopy in biosystems. Chem Phys Chemn 8, 2412-2431 (2007).

20. Yu, G. et al. Polyrotaxane-based supramolecular theranostics. Nat. Commun. 9, 766 (2018).

21. Schroeder, T. B. H. et al. An electric-eel-inspired soft power source from stacked hydrogels. Nature 552, 214-218 (2017).

22. Wood, C. S. \& Stevens, M. M. Improving the image of nanoparticles. Nature 539, 505-506 (2016).

23. Chen, L.-Y. et al. Processing and properties of magnesium containing a dense uniform dispersion of nanoparticles. Nature 528, 539-543 (2015).

24. Zabow, G., Dodd, S. J. \& Koretsky, A. P. Shape-changing magnetic assemblies as high-sensitivity NMR-readable nanoprobes. Nature 520, 73-77 (2015).

25. Morimoto, N. et al. Fast and effective mitochondrial delivery of $\omega$-Rhodamine-B-polysulfobetaine-PEG copolymers. Sci. Rep. 8, 1128 (2018).

26. Morimoto, N. et al. Membrane translocation and organelle-selective delivery steered by polymeric zwitterionic nanospheres. Biomacromolecules 17, 1523-1535 (2016).

27. Takahashi, M. \& Ishikawa, Y. Translational vibrations between chains of hydrogen-bonded molecules in solid-state aspirin form I. Chem. Phys. Lett. 576, 21-25 (2013).

28. Zeitler, J. A. et al. Drug hydrate systems and dehydration processes studied by terahertz pulsed spectroscopy. Int. J. Pharm. 334, 78-84 (2007).

29. Markelz, A., Whitmire, S., Hillebrecht, J. \& Birge, R. THz time domain spectroscopy of biomolecular conformational modes. Phys. Med. Biol. 47, 3797-3805 (2002).

30. Takahashi, M., Okamura, N., Fan, X., Shirakawa, H. \& Minamide, H. Temperature dependence in the terahertz spectrum of nicotinamide: Anharmonicity and hydrogen-bonded network. J. Phys. Chem. A 121, 2558-2564 (2017).

31. Takahashi, M. Flat building blocks for flat silicene. Sci. Rep. 7, 10855 (2017).

32. Takahashi, M., Okamura, N., Ding, X., Shirakawa, H. \& Minamide, H. Intermolecular hydrogen bond stretching vibrations observed in terahertz spectra of crystalline vitamins. CrystEngComm 20, 1960-1969 (2018).

33. Takahashi, M. Terahertz vibrations and hydrogen-bonded networks in crystals. Crystals 4, 74-103 (2014).

34. Araki, K. et al. Synchrotron FTIR microspectroscopy for structural analysis of Lewy bodies in the brain of Parkinson's disease patients. Sci. Rep. 5, 17625 (2015).

35. Jeffrey, G. A. An introduction to hydrogen bonding (Oxford University Press, 1997).

36. Tomasi, J., Mennucci, B. \& Cammi, R. Quantum mechanical continuum solvation models. Chem. Rev. 105, 2999-3093 (2005)

37. Steiner, T. Donor and acceptor strengths in C-H... O hydrogen bonds quantified from crystallographic data of small solvent molecules. New J. Chem. 1099-1103 (1998).

38. Bondi, A. Van der Waals volumes and radii. J. Phys. Chem. 68, 441-451 (1964).

39. Takahashi, M. \& Ishikawa, Y. Terahertz vibrations of crystalline $\alpha$-D-glucose and the spectral change in mutual transitions between the anhydride and monohydrate. Chem. Phys. Lett. 642, 29-34 (2015).

40. Takahashi, M., Ishikawa, Y. \& Ito, H. The dispersion correction and weak-hydrogen-bond network in low-frequency vibration of solid-state salicylic acid. Chem. Phys. Lett. 531, 98-104 (2012).

41. Takahashi, M., Ishikawa, Y., Nishizawa, J.-I. \& Ito, H. Low-frequency vibrational modes of riboflavin and related compounds. Chem. Phys. Lett. 401, 475-482 (2005).

42. Hoshina, H. et al. Higher order conformation of poly(3-hydroxyalkanoates) studied by terahertz time-domain spectroscopy. Appl. Phys. Lett. 96, 101904 (2010).

43. Gaussian 09, Revision D.01 and Gaussian 16, Revision B.01, Frisch, M. J. et al. Gaussian, Inc., (Wallingford CT, 2013 and 2016).

44. Becke, A. D. Density-functional thermochemistry. III. The role of exact exchange. J. Chem. Phys. 98, 5648-5652 (1993).

45. Lee, C., Yang, W. \& Parr, R. G. Development of the Colle-Salvetti correlation-energy formula into a functional of the electron density. Phys. Rev. B 37, 785-789 (1988). 
46. Miehlich, B., Savin, A., Stoll, H. \& Preuss, H. Results obtained with the correlation energy density functionals of Becke and Lee, Yang and Parr. Chem. Phys. Lett. 157, 200-206 (1989).

47. Dunning, T. H. Jr. Gaussian basis sets for use in correlated molecular calculations. I. The atoms boron through neon and hydrogen. J. Chem. Phys. 90, 1007-1023 (1989).

48. Kendall, R. A., Dunning, T. H. Jr. \& Harrison, R. J. Electron affinities of the first-row atoms revisited. Systematic basis sets and wave functions. J. Chem. Phys. 96, 6796-6806 (1992).

49. Woon, D. E. \& Dunning, T. H. Jr. Gaussian basis sets for use in correlated molecular calculations. III. The atoms aluminum through argon. J. Chem. Phys. 98, 1358-1371 (1993).

50. Peterson, K. A., Woon, D. E. \& Dunning, T. H. Jr. Benchmark calculations with correlated molecular wave functions. IV. The classical barrier height of the $\mathrm{H}+\mathrm{H}_{2} \rightarrow \mathrm{H}_{2}+\mathrm{H}$ reaction. J. Chem. Phys. 100, 7410-7415 (1994).

51. Wilson, A. K., Mourik, T. \& Dunning, T. H. Jr. Gaussian basis sets for use in correlated molecular calculations. VI. Sextuple zeta correlation consistent basis sets for boron through neon. J. Mol. Struct. (Theochem) 388, 339-349 (1996).

52. Austin, A. et al. A density functional with spherical atom dispersion terms. J. Chem. Theory Comput. 8, 4989-5007 (2012).

53. Rappé, A. K., Casewit, C. J., Colwell, K. S., Goddard, W. A. III. \& Skiff, W. M. UFF, a full periodic table force field for molecular mechanics and molecular dynamics simulations. J. Am. Chem. Soc. 114, 10024-10035 (1992).

54. Fiedler, J. et al. Effective polarizability models. J. Phys. Chem. A 121, 9742-9751 (2017).

55. Fiedler, J. et al. Dispersion forces in inhomogeneous planarly layered media: A one-dimensional model for effective polarizabilities. Phys. Rev. A 99, 062512 (2019).

56. Grimme, S., Antony, J., Ehrlich, S. \& Krieg, H. A consistent and accurate ab initio parametrization of density functional dispersion correction (DFT-D) for the 94 elements H-Pu. J. Chem. Phys. 132, 154104 (2010).

\section{Acknowledgements}

This research was supported by a (JSPS) Grand-in Aid for Scientific Research (C) (Grant No. 17K05825) and Ube foundation academic incentive grant. The authors acknowledge the Center for Computational Materials Science of IMR, Tohoku University. The synchrotron radiation measurements were performed at the BL43IR of SPring-8 with the approval of the Japan Synchrotron Radiation Research Institute (JASRI) (Proposal No. 2016B1321, 2017A1354, and 2018A1198).

\section{Author Contributions}

M.T. directed the research and wrote the manuscript. M.T. performed DFT calculations and sample settings of synchrotron FTIR microspectroscopy and THz-TDS. M.T. and Y.I measured the synchrotron FTIR microspectra. H.M. manipulated THz-TDS and measured spectra. M.S and N.M. proposed the measurements of DMAPS. All authors made intensive discussions and approved the manuscript.

\section{Additional Information}

Supplementary information accompanies this paper at https://doi.org/10.1038/s41598-019-49352-1.

Competing Interests: The authors declare no competing interests.

Publisher's note: Springer Nature remains neutral with regard to jurisdictional claims in published maps and institutional affiliations.

(c) (i) Open Access This article is licensed under a Creative Commons Attribution 4.0 International License, which permits use, sharing, adaptation, distribution and reproduction in any medium or format, as long as you give appropriate credit to the original author(s) and the source, provide a link to the Creative Commons license, and indicate if changes were made. The images or other third party material in this article are included in the article's Creative Commons license, unless indicated otherwise in a credit line to the material. If material is not included in the article's Creative Commons license and your intended use is not permitted by statutory regulation or exceeds the permitted use, you will need to obtain permission directly from the copyright holder. To view a copy of this license, visit http://creativecommons.org/licenses/by/4.0/.

(C) The Author(s) 2019 